INPLASY

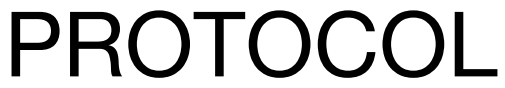

To cite: Liu et al. A systematic review and meta-analysis of impact of statins use on the clinical outcome of patients with coronary spasm. Inplasy protocol 202180066. doi: 10.37766/inplasy2021.8.0066

Received: 16 August 2021

Published: 16 August 2021

Corresponding author: Liu Qilin

1492828910@qq.com

Author Affiliation:

The 305th Hospital of the

People's Liberation Army.

Support: Hospital fund.

Review Stage at time of this submission: The review has not yet started.

Conflicts of interest:

None declared.

\section{A systematic review and meta- analysis of impact of statins use on the clinical outcome of patients with coronary spasm}

\author{
Liu, QL1; Zhang, T2; Xue, Y3; Du, DY4; Liu, Y5; Li, YT6.
}

Review question / Objective: The purpose of this metaanalysis study is to evaluate whether statins affect the clinical outcome of patients with coronary spasm.

Condition being studied: At present, whether statins have a beneficial effect on patients with coronary spasm is controversial in clinical practice, and because the main cause of coronary spasm is endothelial dysfunction, and statins have been shown to repair vascular endothelial function, but for patients without severe In patients with coronary artery spasm with coronary artery stenosis, it is not clear whether statins have a long-term beneficial effect.

INPLASY registration number: This protocol was registered with the International Platform of Registered Systematic Review and Meta-Analysis Protocols (INPLASY) on 16 August 2021 and was last updated on 16 August 2021 (registration number INPLASY202180066).

\section{INTRODUCTION}

Review question / Objective: The purpose of this meta-analysis study is to evaluate whether statins affect the clinical outcome of patients with coronary spasm.
Condition being studied: At present, whether statins have a beneficial effect on patients with coronary spasm is controversial in clinical practice, and because the main cause of coronary spasm 
is endothelial dysfunction, and statins have been shown to repair vascular endothelial function, but for patients without severe In patients with coronary artery spasm with coronary artery stenosis, it is not clear whether statins have a long-term beneficial effect.

\section{METHODS}

Participant or population: Patients with coronary spasm.

Intervention: Statins was the main intervention.

Comparator: Control group didn't treat with statins.

Study designs to be included: Prospective study.

Eligibility criteria: 1. Repeated reports; 2. Defects in research design; 3. Incomplete data; 4. Outcome effects are not MACE.

Information sources: Pubmed, google scholar, embase, cochrane Library.

Main outcome(s): 1. Cardiogenic death; 2. Myocardial infarction; 3. Non-cardiogenic death.

Additional outcome(s): 1. Severe arrhythmia; 2. Recurrent angina pectoris; 3. Revascularization.

Quality assessment / Risk of bias analysis: Newcastle-Ottawa Scale (NOS).

Strategy of data synthesis: We will search articles in four electronic database include pubmed, google scholar, embase, cochrane library. The search string will be built as follows:The search string will be built as follows: (vasospastic angina OR coronary vasospasms OR vasospasm OR variant angina OR Prinzmetal's variant angina OR spastic coronary angina OR coronary artery spasm) AND (statin).The electronic database search will be supplemented by a manual search of the reference lists of included articles.
Subgroup analysis: We will analyze the effects of statins on the subgroup of cardiogenic death, acute myocardial infarction, and revascularization.

Sensitivity analysis: We will use Stata software for sensitivity analysis.

Country(ies) involved: China.

Keywords: coronary spasm; meta-analysis.

Contributions of each author:

Author 1 - Liu Qilin.

Author 2 - Zhang Tao.

Author 3 - Xue Yi.

Author 4 - Du Dayong.

Author 5 - Liu Yang.

Author 6 - Li Yuntian. 